\title{
Nucleon effects on the photon dispersion relations in matter
}

\author{
Juan Carlos D'Olivo \\ Instituto de Ciencias Nucleares \\ Universidad Nacional Autónoma de México \\ Apartado Postal 70-543, 04510 México, D.F., México \\ José F. Nieves \\ Laboratory of Theoretical Physics, Department of Physics, P.O. Box 23343 \\ University of Puerto Rico, Río Piedras, Puerto Rico 00931-3343
}

(hep-ph/9710305)

\begin{abstract}
We calculate the nucleon contribution to the photon self-energy in a plasma, including the effect of the anomalous magnetic moment of the nucleons. General formulas for the transverse and longitudinal components of the self-energy are obtained and we give explicit results in various limits of physical interest. The formulas are relevant for the study of the photon dispersion relations and the dynamical susceptibility in a nuclear medium such as the core of a supernova, and has implications with regard to the recent suggestion that the Cerenkov process $\nu \rightarrow \nu \gamma$ can take place in such a system.
\end{abstract}

\section{INTRODUCTION}

The methods of Finite Temperature Field Theory (FTFT) [1] have proven to be very useful in the study of the matter effects on the propagation of the elementary particles. In these studies, a central role is played by the inverse propagator, or equivalently, the self-energy of the particle. The latter quantity enters directly in the linear part of the equations of motion for the effective classical field, and therefore determines the modes that can propagate through the medium. In a modern context, this point of view has been stimulated by the influential work of Weldon [2, 4 , who emphasized the convenience of covariant, real-time, calculations. His approach has been applied to investigate the effects of a medium on the properties of photons, gauge fields, and neutrinos, in a variety of physical conditions.

The case of photons occupies an important place within these type of problems. Its applications range from the calculation of cross sections for particle emission by stars, to the determination of the optical properties of solids. Under certain conditions of the matter background, the photon dispersion relation in a plasma can be obtained by a semiclassical approach based on the Maxwell-Boltzmann-Vlasov equation [0]. It seems that the first complete quatumfield-theoretic treatment of the same problem is due to Tsytovich [6]. He generalized earlier work along similar lines 17], obtaining results that are valid under more general conditions than those based on the semiclassical approach. The work of Weldon [2] demonstrated that the real-time formulation of FTFT is well suited to study this physical system in an efficient and transparent way, and can be extended to, for example, non-abelian gauge fields.

For photons that propagate in a hot plasma, the medium typically consists of an electron gas superimposed over a static positive background. It can be shown that the results of the semiclassical calculation are recovered from those of the field-theoretic treatment, by taking the limit in which the photon energy and momentum are small relative to the electron mass. Ordinarily, only the electrons play an important role, and the dynamical effect of the nucleons in the background are ignored. This is justified within the context of a semiclassical approach. In fact, the contributions of the ions and electrons to the dielectric constant have similar form. Since the plasma frequency associated with a certain particle is inversely proportional to its mass, the contribution of the ions turns out to be negligible, at least in the most common situation where all species share a common temperature [8].

In a quantum-theoretic approach, however, the electromagnetic couplings of the nucleons must include their anomalous magnetic moments. For the case in which the nucleons constitute a degenerate non-relativistic gas, a standard treatment yields the Pauli formula for the paramagnetic spin susceptibility, in the limit of zero photon frequency (static limit) [9. Nevertheless, a general treatment of the effect in the photon dispersion relation due to the anomalous electromagmetic couplings of the nucleons seems to be lacking in the literature. Such a study has applications in some astrophysical contexts, where systems with dense nuclear matter need to be considered. For example, it has been recently pointed out by Mohanty and Samal [10] that, in a supernova core, the photon dispersion relation receives a contribution from the nucleon magnetic moment which is of the opposite sign to that of the usual electron plasma effect. Under such circumstances, the emission of Cerenkov radiation by neutrinos becomes possible, which can have important implications for the energetics of the system. More recently, Raffelt [11] has noted that the conclusions of Ref. [10] are based on the adoption of the static paramagnetic susceptibility formula mentioned above, in order to 
determine the effect of the nucleons on the photon dispersion relation. However, for a proper account of this effect what is required is the full nucleon contribution to photon self-energy (or, equivalently, to the dielectric constant or the paramagnetic susceptibility) for any value of the photon momentum and not just in the zero-frequency limit. The necessary calculations along these lines are not in the literature, to the best of our knowledge.

Motivated by the above considerations, in the present work we calculate the photon self-energy in a matter background including the contribution from the nucleons, and in particular the effect of their anomalous electromagnetic couplings. The calculation is based on the application of FTFT to this problem and complements the existing calculations of the same quantity for the electron gas alone. The results for the photon self-energy can be equivalently interpreted in terms of the dielectric constant and the susceptibility of the system, and in that way we show that the well known textbooks results for these quantities are reproduced when the appropriate limits are taken. On the other hand, the results we obtain are valid for general conditions of the nucleon gas, whether it is degenerate or not, and also for general values of the photon momentum and not just for some particular limiting values. Therefore, they are relevant and useful in the study of electromagnetic processes ocurring within dense nuclear matter, such as the neutrino Cerenkov radiation in a supernova core mentioned above.

In Section [I] we give the general 1-loop formulas for the generic contribution of a fermion to the photon self-energy, including the effect of a possible magnetic moment coupling of the fermion to the electromagnetic field. There it is shown that the contribution from any given fermion can be written in terms of just three independent functions, which are expressed as integrals over the momentum distribution functions of the fermion. In Section III] explicit formulas are given for various limiting cases of physical interest. The formulas so obtained are used in Section IV to study the photon dispersion relations in a background composed of electrons and nucleons under various conditions, including those corresponding to a degenerate or a classical nucleon background. Although the focus of our calculations is the real part of the self-energy, there we also summarize the results of the calculation of the imaginary part, which determines the damping of the propagating modes. Some details of that calculation are given in Appendix B. Section $\mathrm{V}$ contains our conclusions, and in Appendix A the relation between the self-energy and the dielectric function of macroscopic electrodynamics is briefly reviewed.

\section{PHOTON SELF-ENERGY}

As shown in Ref. [12], the equation of motion for the effective field in the medium, from which the dispersion relations of the propagating modes are determined, is given by

$$
\left[-q^{2} \tilde{g}_{\mu \nu}+\pi_{\mu \nu}^{(e f f)}\right] A^{\nu}=j_{\mu},
$$

where

$$
\tilde{g}_{\mu \nu}=g_{\mu \nu}-\frac{q_{\mu} q_{\nu}}{q^{2}} .
$$

and

$$
\pi_{\mu \nu}^{(e f f)}=\pi_{\mu \nu} \theta(q \cdot u)+\pi_{\nu \mu}^{*} \theta(-q \cdot u) .
$$

In Eq. (2.3) $\theta$ is the step function, $u_{\mu}$ stands for the velocity four-vector of the medium and $\pi_{\mu \nu}$ is determined in terms of the elements of the $2 \times 2$ self-energy matrix in the form

$$
\begin{gathered}
\operatorname{Re} \pi_{\mu \nu}=\operatorname{Re} \pi_{11 \mu \nu} \\
\operatorname{Im} \pi_{\mu \nu}=\tanh \left|\frac{1}{2} \beta q \cdot u\right| \operatorname{Im} \pi_{11 \mu \nu} \\
=\frac{i \pi_{12 \mu \nu} \varepsilon(q \cdot u)}{2 n_{\gamma}(x)}
\end{gathered}
$$

where we have defined

$$
\begin{aligned}
\operatorname{Re} \pi_{\mu \nu} & =\frac{1}{2}\left(\pi_{\mu \nu}+\pi_{\nu \mu}^{*}\right) \\
\operatorname{Im} \pi_{\mu \nu} & =\frac{1}{2 i}\left(\pi_{\mu \nu}-\pi_{\nu \mu}^{*}\right),
\end{aligned}
$$


with similar definitions for $\operatorname{Re} \pi_{11 \mu \nu}$ and $\operatorname{Im} \pi_{11 \mu \nu}$. In Eq. 2.5) $\beta$ is the inverse temperature, $\varepsilon(z)=\theta(z)-\theta(-z)$ and

$$
n_{\gamma}(x)=\frac{1}{e^{x}-1}
$$

where, for the photon,

$$
x=\beta q \cdot u .
$$

In our calculations we have adopted $u^{\mu}=(1, \overrightarrow{0})$, so that all the three-dimensional quantities refer to the frame in which the medium is at rest.

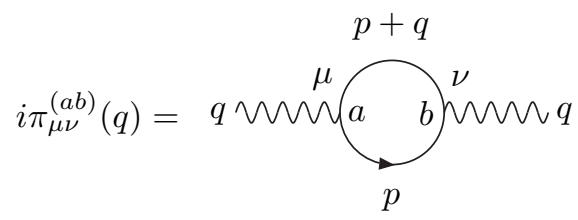

FIG. 1. Diagram for the contribution to the photon self-energy matrix from a generic fermion.

In practical calculations it is much simpler to determine the imaginary part of the self-energy using the second form in Eq. (2.5), and therefore we will determine $\pi_{\mu \nu}^{(e f f)}$ from the formulas

$$
\begin{aligned}
& \operatorname{Re} \pi_{\mu \nu}^{(e f f)}=\operatorname{Re} \pi_{11 \mu \nu} \\
& \operatorname{Im} \pi_{\mu \nu}^{(e f f)}=\frac{i \pi_{12 \mu \nu}}{2 n_{\gamma}(x)} .
\end{aligned}
$$

The various components of the photon self-energy matrix are calculated from the diagram of Fig. 1. For any fermion $f$ in the loop we use the propagator

$$
\begin{aligned}
& S_{F 11}^{(f)}(p)=\left(\not p+m_{f}\right)\left[\frac{1}{p^{2}-m_{f}^{2}+i \epsilon}+2 \pi i \delta\left(p^{2}-m_{f}^{2}\right) \eta_{f}(p)\right] \\
& S_{F 22}^{(f)}(p)=\left(\not p+m_{f}\right)\left[\frac{-1}{p^{2}-m_{f}^{2}-i \epsilon}+2 \pi i \delta\left(p^{2}-m_{f}^{2}\right) \eta_{f}(p)\right] \\
& S_{F 12}^{(f)}(p)=\left(\not p+m_{f}\right) 2 \pi i \delta\left(p^{2}-m_{f}^{2}\right)\left[\eta_{f}(p)-\theta(-p \cdot u)\right] \\
& S_{F 21}^{(f)}(p)=\left(\not p+m_{f}\right) 2 \pi i \delta\left(p^{2}-m_{f}^{2}\right)\left[\eta_{f}(p)-\theta(p \cdot u)\right] .
\end{aligned}
$$

In these formulas

$$
\eta_{f}(p)=\theta(p \cdot u) n_{F}\left(y_{f}\right)+\theta(-p \cdot u) n_{F}\left(-y_{f}\right)
$$

with

$$
n_{F}(y)=\frac{1}{e^{y}+1}
$$

and

$$
y_{f}=\beta p \cdot u-\alpha_{f},
$$

where $\alpha_{f}$ is the fermion chemical potential. 
We consider the calculation of $\pi_{11 \mu \nu}$, from which the real part of the physical self-energy is determined by means of Eq. (2.9). Referring to Fig. 1, the contribution from one fermion in the loop is

$$
i \pi_{11 \mu \nu}^{(f)}=(-1)(-i)^{2} \operatorname{Tr} \int \frac{d^{4} p}{(2 \pi)^{4}} j_{f \mu}^{(e m)}(q) i S_{F 11}^{(f)}(p+q) j_{f \nu}^{(e m)}(-q) i S_{F 11}^{(f)}(p) .
$$

The electromagnetic couplings are defined by writing the matrix element

$$
\left\langle f\left(p^{\prime}\right)\left|J_{\mu}^{(e m)}(0)\right| f(p)\right\rangle=\bar{u}\left(p^{\prime}\right) j_{f \mu}^{(e m)}(q) u(p),
$$

where $q=p-p^{\prime}$ is the momentum of the outgoing photon, $u(p)$ is a Dirac spinor and $j_{f \mu}^{(e m)}(q)$ is the total electromagnetic current of the fermion. For the electron $j_{e \mu}^{(e m)}(q)=e \gamma_{\mu}$, while for the nucleons

$$
\begin{aligned}
& j_{p \mu}^{(e m)}(q)=|e| \gamma_{\mu}-i \kappa_{p} \sigma_{\mu \alpha} q^{\alpha} \\
& j_{n \mu}^{(e m)}(q)=-i \kappa_{n} \sigma_{\mu \alpha} q^{\alpha} .
\end{aligned}
$$

In Eq. 2.16) $\kappa_{n, p}$ are the anomalous part of the nucleon magnetic moments, given by

$$
\begin{gathered}
\kappa_{p}=1.79\left(\frac{|e|}{2 m_{p}}\right), \\
\kappa_{n}=-1.91\left(\frac{|e|}{2 m_{n}}\right),
\end{gathered}
$$

$e$ stands for the electron charge and, as usual, $\sigma_{\mu \nu}=\frac{i}{2}\left[\gamma_{\mu}, \gamma_{\nu}\right]$. For arbitrary values of $q$, Eq. (2.16) does not hold and, in general, we have to parametrize the nucleon electromagnetic current in terms of the momentum-dependent form factors. However, in the applications of interest to us the photon momentum is sufficiently small that the form factors are well approximated by their values at $q^{2}=0$, and therefore Eq. (2.16) is appropriate.

Substituting the formula for $S_{F 11}^{(f)}$ given in Eq. (2.10), $\pi_{11 \mu \nu}^{(f)}$ can be written in the form

$$
\pi_{11 \mu \nu}^{(f)}=\pi_{11 \mu \nu}^{(0)}+\pi_{11 \mu \nu}^{(f)}
$$

where $\pi_{11 \mu \nu}^{(0)}$ is the standard vacuum polarization term, while the background dependent contribution is given by

$$
\begin{aligned}
i \pi_{11 \mu \nu}^{\prime(f)}= & -(2 \pi i) \int \frac{d^{4} p}{(2 \pi)^{4}} \operatorname{Tr}\left[j_{f \mu}^{(e m)}(q)\left(\not p+\not q+m_{f}\right) j_{f \nu}^{(e m)}(-q)\left(\not p+m_{f}\right)\right] \\
& \times\left\{\frac{\eta_{f}(p) \delta\left(p^{2}-m_{f}^{2}\right)}{(p+q)^{2}-m_{f}^{2}+i \epsilon}+\frac{\eta_{f}(p+q) \delta\left((p+q)^{2}-m_{f}^{2}\right)}{p^{2}-m_{f}^{2}+i \epsilon}\right. \\
& \left.+2 \pi i \eta_{f}(p) \eta_{f}(p+q) \delta\left(p^{2}-m_{f}^{2}\right) \delta\left((p+q)^{2}-m_{f}^{2}\right)\right\} .
\end{aligned}
$$

The term in Eq. (2.19) that contains two factors of $\eta_{f}$ contributes only to the imaginary part of the self-energy. We restrict our attention for the moment on the real part, which receives contributions only from the terms linear in $\eta_{f}$, and which we denote by $\operatorname{Re} \pi_{f \mu \nu}^{(e f f)}$. Then, making the change of variables $p+q \rightarrow p$ in the second of these terms in Eq. (2.19) and carrying out the integral over $p_{0}$, we obtain from Eq. 2.9)

$$
\operatorname{Re} \pi_{p \mu \nu}^{(e f f)}=\pi_{\mu \nu}^{(\gamma)}+\pi_{\mu \nu}^{(\sigma)}+\pi_{\mu \nu}^{(\gamma \sigma)},
$$

where

$$
\begin{aligned}
\pi_{\mu \nu}^{(\gamma)} & =-4 e_{f}^{2} \int \frac{d^{3} \mathcal{P}}{(2 \pi)^{3} 2 \mathcal{E}}\left(f_{f}+f_{\bar{f}}\right)\left[\frac{L_{\mu \nu}}{q^{2}+2 p \cdot q}+(q \rightarrow-q)\right] \\
\pi_{\mu \nu}^{(\sigma)} & =-4 \kappa_{f}^{2} \int \frac{d^{3} \mathcal{P}}{(2 \pi)^{3} 2 \mathcal{E}}\left(f_{f}+f_{\bar{f}}\right)\left[\frac{M_{\mu \nu}}{q^{2}+2 p \cdot q}+(q \rightarrow-q)\right] \\
\pi_{\mu \nu}^{(\gamma \sigma)} & =-8\left(e_{f} \kappa_{f} m_{f}\right) q^{2} \tilde{g}_{\mu \nu} \int \frac{d^{3} \mathcal{P}}{(2 \pi)^{3} 2 \mathcal{E}}\left(f_{f}+f_{\bar{f}}\right)\left[\frac{1}{q^{2}+2 p \cdot q}+(q \rightarrow-q)\right] .
\end{aligned}
$$


In these formulas,

$$
\begin{gathered}
L_{\mu \nu}=2 p_{\mu} p_{\nu}+p_{\mu} q_{\nu}+q_{\mu} p_{\nu}-g_{\mu \nu} p \cdot q \\
M_{\mu \nu}=q^{2}\left(2 m_{f}^{2}-p \cdot q\right) \tilde{g}_{\mu \nu}-2(p \cdot q)^{2} g_{\mu \nu}+2(p \cdot q)\left[p_{\mu} q_{\nu}+q_{\mu} p_{\nu}\right]-2 q^{2} p_{\mu} p_{\nu} \\
p^{\mu}=(\mathcal{E}, \overrightarrow{\mathcal{P}}), \quad \mathcal{E}=\sqrt{\overrightarrow{\mathcal{P}}^{2}+m_{f}^{2}}
\end{gathered}
$$

and $f_{f, \bar{f}}$ denote the particle and antiparticle number density distributions given by

$$
f_{f, \bar{f}}=\frac{1}{e^{\beta \mathcal{E} \mp \alpha_{f}}+1}
$$

with the minus(plus) sign holding for the particles(antiparticles), respectively. The integrals in Eq. (2.21) is to be interpreted in the sense of its principal value part.

As we have already mentioned, for the imaginary part of $\pi_{\mu \nu}^{(e f f)}$ it is simpler to calculate $\pi_{12 \mu \nu}$ first and then use the second form of the formula for $\operatorname{Im} \pi_{\mu \nu}^{(e f f)}$ given in Eq. (2.9), instead of calculating $\operatorname{Im} \pi_{11 \mu \nu}$. Nevertheless, we have carried out the calculation in both ways and explicitly verified that they yield the same result for $\pi_{\mu \nu}^{(e f f)}$. The results of that calculation are summarized in Section IVF, and the main steps leading to it are oulined in Appendix B. In the remainder of this paper we focus on the real part of $\pi_{\mu \nu}^{(e f f)}$.

In an isotropic medium, the most general form of the photon self-energy is [2, 13 .

$$
\pi_{\mu \nu}^{(e f f)}=\pi_{T} R_{\mu \nu}+\pi_{L} Q_{\mu \nu}+\pi_{P} P_{\mu \nu}
$$

where

$$
\begin{aligned}
R_{\mu \nu} & =\tilde{g}_{\mu \nu}-Q_{\mu \nu} \\
Q_{\mu \nu} & =\frac{\tilde{u}_{\mu} \tilde{u}_{\nu}}{\tilde{u}^{2}} \\
P_{\mu \nu} & =\frac{i}{\mathcal{Q}} \epsilon_{\mu \nu \alpha \beta} q^{\alpha} u^{\beta},
\end{aligned}
$$

with

$$
\tilde{u}_{\mu} \equiv \tilde{g}_{\mu \nu} u^{\nu}
$$

In general, $\pi_{T, L, P}$ are functions of the scalar variables

$$
\begin{aligned}
& \Omega=q \cdot u \\
& \mathcal{Q}=\sqrt{\Omega^{2}-q^{2}},
\end{aligned}
$$

which have the interpretation of being the photon energy and momentum (in the rest frame of the medium). The conditions under which the function $\pi_{P}$ can have a nonzero value, as well as some of its implications, have been discussed in Ref. [13]. In the present case, it is easily seen that it is zero since all the terms in Eq. (2.21) are symmetric in $\mu, \nu$. The functions $\pi_{T, L}$ are determined by projecting Eq. (2.20) with the tensors $R_{\mu \nu}$ and $Q_{\mu \nu}$. This procedure then leads to

$$
\begin{aligned}
\pi_{\mu \nu}^{(\gamma)} & =-4 e_{f}^{2}\left[\frac{1}{2}\left(A_{f}-\frac{B_{f}}{\tilde{u}^{2}}\right) R_{\mu \nu}+\frac{B_{f}}{\tilde{u}^{2}} Q_{\mu \nu}\right], \\
\pi_{\mu \nu}^{(\sigma)} & =-4 \kappa_{f}^{2}\left[\frac{1}{2}\left(A_{f}^{\prime}-\frac{B_{f}^{\prime}}{\tilde{u}^{2}}\right) R_{\mu \nu}+\frac{B_{f}^{\prime}}{\tilde{u}^{2}} Q_{\mu \nu}\right], \\
\pi_{\mu \nu}^{(\gamma \sigma)} & =-8\left(e_{f} \kappa_{f} m_{f}\right) q^{2} D_{f}\left[R_{\mu \nu}+Q_{\mu \nu}\right]
\end{aligned}
$$

where, 


$$
\begin{aligned}
A_{f} & \equiv \int \frac{d^{3} \mathcal{P}}{(2 \pi)^{3} 2 \mathcal{E}}\left(f_{f}+f_{\bar{f}}\right)\left[\frac{L_{\mu}^{\mu}}{q^{2}+2 p \cdot q}+(q \rightarrow-q)\right], \\
B_{f} & \equiv \int \frac{d^{3} \mathcal{P}}{(2 \pi)^{3} 2 \mathcal{E}}\left(f_{f}+f_{\bar{f}}\right)\left[\frac{u^{\mu} u^{\nu} L_{\mu \nu}}{q^{2}+2 p \cdot q}+(q \rightarrow-q)\right], \\
A_{f}^{\prime} & \equiv \int \frac{d^{3} \mathcal{P}}{(2 \pi)^{3} 2 \mathcal{E}}\left(f_{f}+f_{\bar{f}}\right)\left[\frac{M_{\mu}^{\mu}}{q^{2}+2 p \cdot q}+(q \rightarrow-q)\right], \\
B_{f}^{\prime} & \equiv \int \frac{d^{3} \mathcal{P}}{(2 \pi)^{3} 2 \mathcal{E}}\left(f_{f}+f_{\bar{f}}\right)\left[\frac{u^{\mu} u^{\nu} M_{\mu \nu}}{q^{2}+2 p \cdot q}+(q \rightarrow-q)\right], \\
D_{f} & \equiv \int \frac{d^{3} \mathcal{P}}{(2 \pi)^{3} 2 \mathcal{E}}\left(f_{f}+f_{\bar{f}}\right)\left[\frac{1}{q^{2}+2 p \cdot q}+(q \rightarrow-q)\right] .
\end{aligned}
$$

Substituting here the explicit formulas for $L_{\mu \nu}$ and $M_{\mu \nu}$ given in Eqs. (2.22) and (2.22), after some straightforward algebra we obtain the relations

$$
\begin{aligned}
& A_{f}^{\prime}=q^{2}\left(\frac{1}{2} A_{f}+3 m_{f}^{2} D_{f}\right), \\
& B_{f}^{\prime}=-\mathcal{Q}^{2}\left(\frac{1}{2} A_{f}+m_{f}^{2} D_{f}\right)-q^{2} B_{f},
\end{aligned}
$$

with $A_{f}, B_{f}$ being given by

$$
\begin{aligned}
& A_{f}=\int \frac{d^{3} \mathcal{P}}{(2 \pi)^{3} 2 \mathcal{E}}\left(f_{f}+f_{\bar{f}}\right)\left[\frac{2 m_{f}^{2}-2 p \cdot q}{q^{2}+2 p \cdot q}+(q \rightarrow-q)\right] \\
& B_{f}=\int \frac{d^{3} \mathcal{P}}{(2 \pi)^{3} 2 \mathcal{E}}\left(f_{f}+f_{\bar{f}}\right)\left[\frac{2(p \cdot v)^{2}+2(p \cdot v)(q \cdot v)-p \cdot q}{q^{2}+2 p \cdot q}+(q \rightarrow-q)\right] .
\end{aligned}
$$

For later purposes, it is also useful to note here that, by inspection of the formulas for $D_{f}$ and $A_{f}$ given in Eqs. (2.30) and (2.32) respectively, it follows immediately that

$$
A_{f}=-2 \mathcal{J}_{f}+\left(2 m_{f}^{2}+q^{2}\right) D_{f}
$$

where

$$
\mathcal{J}_{f}=\int \frac{d^{3} \mathcal{P}}{(2 \pi)^{3} 2 \mathcal{E}}\left(f_{f}+f_{\bar{f}}\right)
$$

From Eq. (2.29) we can immediately identify the contribution of any fermion to the transverse and longitudinal part of the self-energy, which we denote by $\pi_{T, L}^{(f)}$. Using Eq. 2.31) they can be expressed in the form

$$
\begin{aligned}
& \operatorname{Re} \pi_{T}^{(f)}=-2 e_{f}^{2}\left(A_{f}+\frac{q^{2}}{\mathcal{Q}^{2}} B_{f}\right)-8 e_{f} \kappa_{f} m_{f} q^{2} D_{f}-2 \kappa_{f}^{2} q^{2}\left[2 m_{f}^{2} D_{f}-\frac{q^{2}}{\mathcal{Q}^{2}} B_{f}\right], \\
& \operatorname{Re} \pi_{L}^{(f)}=4 e_{f}^{2} \frac{q^{2}}{\mathcal{Q}^{2}} B_{f}-8 e_{f} \kappa_{f} m_{f} q^{2} D_{f}-4 \kappa_{f}^{2} q^{2}\left[\frac{1}{2} A_{f}+\frac{q^{2}}{\mathcal{Q}^{2}} B_{f}+m_{f}^{2} D_{f}\right] .
\end{aligned}
$$

In this way, the contribution of any fermion to the photon self-energy is expressed in terms of the three functions $A_{f}, B_{f}, D_{f}$. While the evaluation of these functions is not possible in the general case, some useful results can be obtained by considering special cases. Of particular interest to us are the approximate expressions obtained for small values of $q$, which we analyze in some detail in Section III.

\section{LOW PHOTON-MOMENTUM LIMIT}

The functions $A_{f}, B_{f}, D_{f}$ are the same ones that appear in the calculation of the induced electromagnetic vertex of a neutrino in a matter background, which were analyzed in Refs. 14,15] with considerable detail for various limiting 
values of the photon momentum, and for several conditions of a background gas made of nucleons and electrons.t] The results obtained there are directly applicable here. In this section we expand upon those results further.

In most of the situations of practical interest, the photon momentum is such that $\Omega, \mathcal{Q} \ll m_{p, n}$. In this case, from Eq. (2.33), it follows that

$$
D_{f} \simeq \frac{1}{2 m_{f}^{2}}\left(A_{f}+2 \mathcal{J}_{f}\right) .
$$

Later on we use this equation to evaluate $D_{n, p}$. On the other hand, borrowing the results given in Eqs. (A6) and (A11) of Ref. 15], for small values of q we have

$$
\begin{aligned}
& B_{f}=-\frac{1}{2} \int \frac{d^{3} \mathcal{P}}{(2 \pi)^{3}}\left(\frac{\vec{v}_{\mathcal{P}} \cdot \overrightarrow{\mathcal{Q}}}{\Omega-\vec{v}_{\mathcal{P}} \cdot \overrightarrow{\mathcal{Q}}}\right) \frac{d}{d \mathcal{E}}\left(f_{f}+f_{\bar{f}}\right), \\
& A_{f}=B_{f}+\frac{\Omega}{2} \int \frac{d^{3} \mathcal{P}}{(2 \pi)^{3}}\left(\frac{v_{\mathcal{P}}^{2}}{\Omega-\vec{v}_{\mathcal{P}} \cdot \overrightarrow{\mathcal{Q}}}\right) \frac{d}{d \mathcal{E}}\left(f_{f}+f_{\bar{f}}\right),
\end{aligned}
$$

with $\vec{v}_{\mathcal{P}}=\overrightarrow{\mathcal{P}} / \Omega$ denoting the velocity of the particles in the background. The above formula for $B_{f}$ can be rewritten by multiplying the integrand by the factor

$$
\frac{1}{\Omega}\left(\Omega-\vec{v}_{\mathcal{P}} \cdot \overrightarrow{\mathcal{Q}}+\vec{v}_{\mathcal{P}} \cdot \overrightarrow{\mathcal{Q}}\right) .
$$

The first two terms integrate to zero, while the third one leads to

$$
B_{f}=-\frac{1}{2 \Omega} \int \frac{d^{3} \mathcal{P}}{(2 \pi)^{3}}\left(\frac{\left(\vec{v}_{\mathcal{P}} \cdot \overrightarrow{\mathcal{Q}}\right)^{2}}{\Omega-\vec{v}_{\mathcal{P}} \cdot \overrightarrow{\mathcal{Q}}}\right) \frac{d}{d \mathcal{E}}\left(f_{f}+f_{\bar{f}}\right) .
$$

We should note that expressions in Eq. (3.2) are derived from Eq. (2.32) by expanding the integrands in terms of $q / \mathcal{E}$ and retaining only the terms that are dominant when $q / \mathcal{E} \rightarrow 0$. Therefore, Eq. (3.2) is valid for values of $q$ such that

$$
q /\langle\mathcal{E}\rangle \ll 1
$$

where $\langle\mathcal{E}\rangle$ denotes a typical average energy of the fermions in the gas. Thus, for a non-relativistic gas, Eq. (3.2) holds for $\Omega, \mathcal{Q} \ll m_{f}$. If the gas is extremely relativistic, Eq. (3.2) also holds for $\Omega, \mathcal{Q}>m_{f}$, subject to Eq. (3.5).

For an isotropic fermion distribution, an elementary integration on the angular variables yields

$$
\begin{aligned}
& B_{f}=\frac{1}{4 \pi^{2}} \int_{0}^{\infty} d \mathcal{P} \mathcal{P}^{2} \frac{d}{d \mathcal{E}}\left(f_{f}+f_{\bar{f}}\right)\left(1-\frac{\mathcal{E} \Omega}{2 \mathcal{P} \mathcal{Q}} \ln \left|\frac{\mathcal{E} \Omega+\mathcal{P} \mathcal{Q}}{\mathcal{E} \Omega-\mathcal{P} \mathcal{Q}}\right|\right), \\
& A_{f}=B_{f}+\frac{1}{4 \pi^{2}} \int_{0}^{\infty} d \mathcal{P} \frac{\mathcal{P}^{3}}{\mathcal{E}} \frac{d}{d \mathcal{E}}\left(f_{f}+f_{\bar{f}}\right) \frac{\Omega}{2 \mathcal{Q}} \ln \left|\frac{\mathcal{E} \Omega+\mathcal{P} \mathcal{Q}}{\mathcal{E} \Omega-\mathcal{P} \mathcal{Q}}\right| .
\end{aligned}
$$

Up to this point no assumption has been made regarding the nature of the fermion background. Apart from the restriction on $q$, Eqs. (3.2) and (3.6) hold for a relativistic or non-relativistic gas, whether it is degenerate or not. Accordingly, Eq. (3.6) serves as a convenient starting point to find the dispersion relations of photons that propagate through an isotropic medium, provided Eq. (3.5) is verified. In what follows, we present the explicit results for several important physical situations.

\section{A. Degenerate gas}

As functions of the energy, the distribution for a degenerate gas and its derivative are given by

$$
\begin{array}{r}
f_{f}=\theta\left(\mathcal{E}_{F f}-\mathcal{E}\right), \\
\frac{d f_{f}}{d \mathcal{E}}=-\delta\left(\mathcal{E}_{F f}-\mathcal{E}\right) .
\end{array}
$$

\footnotetext{
${ }^{1}$ The quantities denoted by $A_{e}, B_{e}$ in the present paper were denoted by $A, B$ in Refs. 114, 15 .
} 
As a consequence, the integrations needed to determine $A_{f}, B_{f}, \mathcal{J}_{f}$ become very simple. The results can be expressed in the form

$$
\begin{aligned}
& \mathcal{J}_{f}=\frac{m_{f} \mathcal{P}_{F f} \gamma_{f}}{8 \pi^{2}}\left[1-\frac{1}{2 \gamma_{f}^{2} v_{F f}} \log \left(\frac{1+v_{F f}}{1-v_{F f}}\right)\right], \\
& B_{f}=\frac{m_{f} \mathcal{P}_{F f} \gamma_{f}}{4 \pi^{2}}\left[-1+\frac{1}{2} z_{f} \log \left|\frac{1+z_{f}}{1-z_{f}}\right|\right], \\
& A_{f}=\frac{m_{f} \mathcal{P}_{F f} \gamma_{f}}{4 \pi^{2}}\left[-1+\frac{1}{2 \gamma_{f}^{2}} z_{f} \log \left|\frac{1+z_{f}}{1-z_{f}}\right|\right],
\end{aligned}
$$

where

$$
\begin{aligned}
\gamma_{f} & =\frac{1}{\sqrt{1-v_{F f}^{2}}}, \\
z_{f} & =\frac{\Omega}{v_{F f} \mathcal{Q}},
\end{aligned}
$$

with $v_{F f}=\mathcal{P}_{F f} / \mathcal{E}_{F f}$ being the Fermi velocity of the gas. Let us note that

$$
A_{f}(\Omega=\mathcal{Q})=-2 \mathcal{J}_{f},
$$

which is valid in general, for any distribution function and any kinematic regime of the gas, as can be easily checked from Eqs. 2.32) and (2.34).

\section{B. Relativistic gas}

If the fermions are ultrarelativistic, then $\mathcal{E} \simeq \mathcal{P}$ and Eq. (3.6) reduces to

$$
\begin{aligned}
& B_{f}=-3 \omega_{0 f}^{2}\left(1-\frac{\Omega}{2 \mathcal{Q}} \ln \left|\frac{\Omega+\mathcal{Q}}{\Omega-\mathcal{Q}}\right|\right), \\
& A_{f}=-3 \omega_{0 f}^{2} .
\end{aligned}
$$

The quantity $\omega_{0 f}$ is given by

$$
\omega_{0 f}^{2}=\int \frac{d^{3} \mathcal{P}}{(2 \pi)^{3} 2 \mathcal{E}}\left(f_{f}+f_{\bar{f}}\right)\left[1-\frac{\mathcal{P}^{2}}{3 \mathcal{E}^{2}}\right],
$$

and in Eq. (3.11) we have used its limiting form

$$
\omega_{0 f}^{2}=\frac{1}{6 \pi^{2}} \int_{0}^{\infty} d \mathcal{P} \mathcal{P}\left(f_{f}+f_{\bar{f}}\right),
$$

for $\mathcal{E}=\mathcal{P}$. In terms of Eq. (3.13) $\mathcal{J}_{f}$ becomes

$$
\mathcal{J}_{f}=\frac{3}{2} \omega_{0 f}^{2} .
$$

The remaining integral in Eq. (3.13) cannot be performed without specifying the distribution function. For a classical (relativistic) gas $f_{f}=\exp \left(-\beta_{f} \mathcal{P}+\alpha_{f}\right)$ and

$$
\omega_{0 f}^{2}=\frac{\beta_{f}}{12} n_{f},
$$

while for a degenerate gas the integration over $\mathcal{P}$ yields

$$
\omega_{0 f}^{2}=\frac{1}{12}\left(\frac{3 n_{f}}{\pi}\right)^{2 / 3}=\frac{\mathcal{P}_{F f}^{2}}{12 \pi^{2}} .
$$

The formulas for $A_{f}$ and $B_{f}$ given in Eq. (3.11), with $\omega_{0 f}^{2}$ determined by Eq. (3.16), coincide with the relativistic limit $\left(\mathcal{E}_{F} \simeq \mathcal{P}_{F}\right)$ of Eq. (3.8), as it should be. 


\section{Non-relativistic, non-degenerate gas}

The integrals cannot be carried out explicitly in this case. However, some useful approximate results can be obtained by considering the two regions $\Omega \gg \bar{v}_{f} \mathcal{Q}$ and $\Omega \ll \bar{v}_{f} \mathcal{Q}$ separately, where

$$
\bar{v}_{f}^{2} \equiv \frac{1}{\beta_{f} m_{f}},
$$

We have denoted the inverse temperature of the gas by $\beta_{f}$ in order to allow for the possibility that different components of the background may be at different temperatures.

The results that we have obtained in this way can be written in the form

$$
\begin{aligned}
& B_{f}=\frac{\beta_{f} n_{f}}{4} \begin{cases}-1+\bar{z}_{f}^{2} & \left(\text { for } \bar{z}_{f} \ll 1\right) \\
\frac{1}{\bar{z}_{f}^{2}}+\frac{3}{\bar{z}_{f}^{4}} & \left(\text { for } \bar{z}_{f} \gg 1\right),\end{cases} \\
& A_{f}=\frac{\beta_{f} n_{f}}{4} \begin{cases}-1+\bar{z}_{f}^{2}-\bar{v}_{f}^{2} \bar{z}_{f}^{2} & \left(\text { for } \bar{z}_{f} \ll 1\right) \\
\frac{1}{\bar{z}_{f}^{2}}+\frac{3}{\bar{z}_{f}^{4}}-\bar{v}_{f}^{2}\left(3+\frac{5}{\bar{z}_{f}^{2}}\right) & \left(\text { for } \bar{z}_{f} \gg 1\right),\end{cases} \\
& \mathcal{J}_{f}=\frac{n_{f}}{4 m_{f}},
\end{aligned}
$$

with $\bar{v}_{f}$ as defined in Eq. (3.17), and

$$
\bar{z}_{f} \equiv \frac{\Omega}{\bar{v}_{f} \mathcal{Q}} .
$$

As an illustration of how we have proceeded, let us consider in some detail the calculation of $B_{f}$. For a classical non-relativistic gas,

$$
\frac{d f_{f}}{d \mathcal{E}}=-\beta_{f} f_{f}
$$

and from Eq. (3.2)

$$
B_{f}=-\frac{\beta_{f} n_{f}}{4}+\frac{\Omega \beta_{f} m_{f}}{4 \pi^{2} \mathcal{Q}} \int_{0}^{\infty} d \mathcal{P} \mathcal{P} f_{f} \log \left|\frac{1+\xi}{1-\xi}\right|
$$

where

$$
\xi \equiv \frac{\Omega}{v_{\mathcal{P}} \mathcal{Q}}
$$

For the low frequency region, we proceed by putting

$$
\log \left|\frac{1+\xi}{1-\xi}\right| \simeq 2 \xi
$$

and then using the result

$$
\int_{0}^{\infty} d \mathcal{P} f_{f}=\frac{\pi^{2} \beta_{f} n_{f}}{m_{f}}
$$

in Eq. (3.23). In this way the result for $\Omega \ll \bar{v}_{f} \mathcal{Q}$ given in Eq. (3.18) follows. For the high frequency region, we put

$$
\log \left|\frac{1+\xi}{1-\xi}\right| \simeq \frac{2}{\xi}\left(1+\frac{1}{3 \xi^{2}}+\frac{1}{5 \xi^{4}}\right),
$$

and then use, in addition to Eq. (3.26), the results 


$$
\begin{aligned}
& \int_{0}^{\infty} d \mathcal{P} \mathcal{P}^{2} f_{f}=\pi^{2} n_{f}, \\
& \int_{0}^{\infty} d \mathcal{P} \mathcal{P}^{4} f_{f}=\frac{3 \pi^{2} m_{f} n_{f}}{\beta_{f}} \\
& \int_{0}^{\infty} d \mathcal{P} \mathcal{P}^{6} f_{f}=\frac{15 \pi^{2} m_{f}^{2} n_{f}}{\beta_{f}^{2}} .
\end{aligned}
$$

in Eq. (3.23).

Similarly for $A_{f}$, following a similar procedure it is easy to show from Eq. (3.2) that

$$
A_{f}-B_{f}=-\frac{n_{f}}{4 m_{f}} \begin{cases}\left(\frac{\Omega}{\bar{v}_{f} \mathcal{Q}}\right)^{2}, & \left(\text { for } \Omega \ll \bar{v}_{f} \mathcal{Q}\right) \\ 3+5\left(\frac{\bar{v}_{f} \mathcal{Q}}{\Omega}\right)^{2} & \left(\text { for } \Omega \gg \bar{v}_{f} \mathcal{Q}\right)\end{cases}
$$

Therefore, comparing with the formula for $B_{f}$ in Eq. (3.18), we arrive at Eq. (3.19).

\section{DISCUSSION AND APPLICATIONS}

In general, the dispersion relations are obtained by solving Eq. (2.1) with $j_{\mu}=0$ which, using the decomposition in Eq. (2.25) and the fact that $\pi_{P}=0$ in the present context, is equivalent to solve

$$
\left[R_{\mu \nu}\left(-q^{2}+\pi_{T}\right)+Q_{\mu \nu}\left(-q^{2}+\pi_{L}\right)\right] A^{\nu}=0 .
$$

Since $R_{\mu \nu}$ and $Q_{\mu \nu}$ are orthogonal, the solutions to this equation exist only for $q=\left(\omega_{T, L}(\mathcal{Q}), \overrightarrow{\mathcal{Q}}\right)$, where $\omega_{T, L}(\mathcal{Q})$ satisfy

$$
\begin{aligned}
\omega_{T}^{2}-\mathcal{Q}^{2} & =\pi_{T}\left(\omega_{T}, \mathcal{Q}\right), \\
\omega_{L}^{2}-\mathcal{Q}^{2} & =\pi_{L}\left(\omega_{L}, \mathcal{Q}\right) .
\end{aligned}
$$

In general, $\omega_{T, L}$ are complex functions of $\mathcal{Q}$ and can be written in the form $\omega_{T, L}=\Omega_{T, L}-i \gamma_{T, L} / 2$. The quantities $\Omega_{T, L}$ and $\gamma_{T, L}$ are real and have the interpretation of being the dispersion relation and damping rate of the propagating mode, respectively. Retaining terms that are at most linear in $\gamma_{T, L}$ it follows that

$$
\begin{aligned}
\Omega_{T, L}^{2}-\mathcal{Q}^{2} & =\operatorname{Re} \pi_{T, L}\left(\Omega_{T, L}, \mathcal{Q}\right), \\
\frac{\gamma_{T, L}}{2} & =-\left[\frac{\operatorname{Im} \pi_{T, L}}{\frac{\partial}{\partial \omega}\left(\omega^{2}-\operatorname{Re} \pi_{T, L}\right)}\right]_{\omega=\Omega_{T, L}}
\end{aligned}
$$

For a background containing several fermions species,

$$
\operatorname{Re} \pi_{T, L}=\sum_{f} \operatorname{Re} \pi_{T, L}^{(f)}
$$

Each individual contribution in Eq. (4.4) can be immediately identified from Eq. (2.29). Thus, for the electron component we set $\kappa_{e}=0$ while for the neutron $e_{n}=0$. The corresponding expressions for the proton are more complicated due to the presence of two terms in $j_{p \mu}^{(\mathrm{em})}$.

The explicit formulas given in Section III allow us to study in some detail the photon self-energy in a nuclear medium, provided of course that the physical conditions are such that the approximations and idealizations that led to them are valid in the particular context in which they are being applied. As an illustration, we consider some possible situations. 


\section{A. Electron background}

Although the results for this case are well known, we briefly review them here in a form that will be useful for our later purposes. Setting $\kappa_{e}=0$ in Eq. (2.35),

$$
\begin{aligned}
& \operatorname{Re} \pi_{T}^{(e)}=-2 e^{2}\left(A_{e}+\frac{q^{2}}{\mathcal{Q}^{2}} B_{e}\right), \\
& \operatorname{Re} \pi_{L}^{(e)}=4 e^{2} \frac{q^{2}}{\mathcal{Q}^{2}} B_{e} .
\end{aligned}
$$

The functions $A_{e}$ and $B_{e}$ are in given in general by Eq. (2.32) and, in the low $q$ regime, by Eq. (3.2). By specializing these formulas further to the zero frequency (static) limit or zero momentum (long wavelength) limit, we obtain explicit formulas for them, given explicitly in Refs. [14,15], which are useful in practical applications. For example, from Eq. (3.9) of Ref. [15] we have

$$
\begin{aligned}
& A_{e}(\Omega, \mathcal{Q} \rightarrow 0)=-3 \omega_{0 e}^{2}, \\
& B_{e}(\Omega, \mathcal{Q} \rightarrow 0)=\frac{\mathcal{Q}^{2} \omega_{0 e}^{2}}{\Omega^{2}} .
\end{aligned}
$$

Substituting Eq. (4.6) into Eq. (4.5) we then obtain

$$
\operatorname{Re} \pi_{T, L}^{(e)}(\Omega, 0)=4 e^{2} \omega_{0 e}^{2},
$$

which in turns implies the well known result that the longitudinal and transverse permitivity have the same long wavelength limit

$$
\epsilon_{t, l}(\Omega, 0)=1-\frac{4 e^{2} \omega_{0 e}^{2}}{\Omega^{2}} .
$$

Eq. (4.7), or equivalently 4.8, implies the well known expression for the electron contribution to the plasma frequency, namely

$$
\Omega_{e}=\sqrt{4 e^{2} \omega_{0 e}^{2}}
$$

defined as the value $\Omega_{T, L}(0)$ of the dispersion relation in the limit $\mathcal{Q} \rightarrow 0$. In particular, if the electrons are nonrelativistic, then $\omega_{0 e}^{2}=n_{e} / 4 m_{e}$, as is immediately seen from Eq. (3.12), and $\Omega_{e}=\sqrt{e^{2} n_{e} / m_{e}}$. In the relativistic regime, $\omega_{0 e}^{2}$ is given by Eqs. (3.15) and (3.16).

For values of $\mathcal{Q} \neq 0$, using Eqs. (3.2) and (3.4) in Eq. (4.5) we obtain

$$
\begin{aligned}
& \operatorname{Re} \pi_{T}^{(e)}=-e^{2} \Omega \int \frac{d^{3} \mathcal{P}}{(2 \pi)^{3}}\left(\frac{\vec{v}_{\perp} \cdot \vec{v}_{\mathcal{P}}}{\Omega-\vec{v}_{\mathcal{P}} \cdot \overrightarrow{\mathcal{Q}}}\right) \frac{d}{d \mathcal{E}}\left(f_{e}+f_{\bar{e}}\right), \\
& \operatorname{Re} \pi_{L}^{(e)}=-\frac{2 e^{2} q^{2}}{\Omega \mathcal{Q}^{2}} \int \frac{d^{3} \mathcal{P}}{(2 \pi)^{3}}\left(\frac{\left(\vec{v}_{\mathcal{P}} \cdot \overrightarrow{\mathcal{Q}}\right)^{2}}{\Omega-\vec{v}_{\mathcal{P}} \cdot \overrightarrow{\mathcal{Q}}}\right) \frac{d}{d \mathcal{E}}\left(f_{e}+f_{\bar{e}}\right),
\end{aligned}
$$

where

$$
\vec{v}_{\perp}=\vec{v}_{\mathcal{P}}-\left(\vec{v}_{\mathcal{P}} \cdot \hat{\mathcal{Q}}\right) \hat{\mathcal{Q}}
$$

Substituting Eq. (4.12) into Eq. A4) we obtain the explicit formulas for the transverse and longitudinal components $\epsilon_{t, l}$ of the dielectric constant, that coincide precisely with those obtained by the semiclassical approach based on the Boltzman equation [16]. For the particular case of an ultrarelativistic electron gas, using the result in Eq. (3.11) we obtain

$$
\begin{aligned}
& \operatorname{Re} \pi_{T}^{(e)}=3 e^{2} \omega_{0 e}^{2} \frac{\Omega}{\mathcal{Q}}\left(\frac{2 \Omega}{\mathcal{Q}}-\frac{q^{2}}{\mathcal{Q}^{2}} \ln \left|\frac{\Omega+\mathcal{Q}}{\Omega-\mathcal{Q}}\right|\right), \\
& \operatorname{Re} \pi_{L}^{(e)}=-12 e^{2} \omega_{0 e}^{2} \frac{q^{2}}{\mathcal{Q}^{2}}\left(1-\frac{\Omega}{2 \mathcal{Q}} \ln \left|\frac{\Omega+\mathcal{Q}}{\Omega-\mathcal{Q}}\right|\right) .
\end{aligned}
$$

Another useful limit corresponds to set $m_{e} \rightarrow 0$ in Eq. (2.32). The formulas obtained for $\operatorname{Re} \pi_{T, L}^{(e)}$ by substituting the expressions so obtained for $A_{e}$ and $B_{e}$ into Eq. 4.5), coincide with those given by Weldon [2]. Those formulas are valid in the regime $T, q \gg m_{e}$, and neglecting the terms of order $q / T$ they coincide precisely with the result given in Eq. (4.12). In contrast, the formulas given in Eq. (3.2) and the corresponding expressions for $\operatorname{Re} \pi_{T, L}^{(e)}$ obtained from Eq. (4.5) are valid for $q \ll\langle\mathcal{E}\rangle$, but they hold whether $T$ is larger or smaller than $m_{e}$. 


\section{B. Degenerate neutron gas}

The neutron contribution to the photon self-energy is given by setting $e_{n}=0$ in Eq. (2.35). Remembering Eq. (3.1) and using Eq. (3.8), we obtain for this case

$$
\begin{aligned}
& \operatorname{Re} \pi_{T}^{(n)}=q^{2} \chi_{0}^{(n)} \gamma_{n}\left\{\frac{1}{2 \gamma_{n}^{2}}\left[\frac{1}{2 v_{F n}} \log \left(\frac{1+v_{F n}}{1-v_{F n}}\right)-1\right]+\left[1-\frac{1}{2} v_{F n}^{2}\left(1+z_{n}^{2}\right)\right]\left[1-\frac{1}{2} z_{n} \log \left|\frac{1+z_{n}}{1-z_{n}}\right|\right]\right\} \\
& \operatorname{Re} \pi_{L}^{(n)}=q^{2} \chi_{0}^{(n)} \gamma_{n} v_{F n}^{2}\left\{\frac{1}{2 v_{F n}^{2}}\left[\frac{1}{2 v_{F n} \gamma_{n}^{2}} \log \left(\frac{1+v_{F n}}{1-v_{F n}}\right)-1\right]+z_{n}^{2}+\frac{1}{2} z_{n}\left(1-z_{n}^{2}\right) \log \left|\frac{1+z_{n}}{1-z_{n}}\right|\right\}
\end{aligned}
$$

where we have defined

$$
\chi_{0}^{(n)} \equiv \frac{\kappa_{n}^{2} m_{n} \mathcal{P}_{F n}}{\pi^{2}} .
$$

We emphasize that the above formulas are valid for all values of $q$ subject only to $\Omega, \mathcal{Q} \ll m_{n}$, and also they hold in the non-relativistic as well as the relativistic case. However, in the non-relativistic limit, they reduce to

$$
\begin{aligned}
& \operatorname{Re} \pi_{T}^{(n)}=q^{2} \chi_{0}^{(n)} f_{T}\left(z_{n}\right) \\
& \operatorname{Re} \pi_{L}^{(n)}=q^{2} \chi_{0}^{(n)} v_{F n}^{2} f_{L}\left(z_{n}\right),
\end{aligned}
$$

where

$$
\begin{aligned}
& f_{T}(z)=\frac{1}{6} v_{F}^{2}+\left[1-\frac{1}{2} v_{F}^{2} z^{2}\right]\left[1-\frac{1}{2} z \log \left|\frac{1+z}{1-z}\right|\right] \\
& f_{L}(z)=-\frac{1}{3}+z^{2}+\frac{1}{2} z\left(1-z^{2}\right) \log \left|\frac{1+z}{1-z}\right|
\end{aligned}
$$

In the limits $\Omega \rightarrow 0$ or $\mathcal{Q} \rightarrow 0$ we obtain from Eq. (4.15) the limiting values

$$
\begin{aligned}
\operatorname{Re} \pi_{T}^{(n)}(0, \mathcal{Q}) & =-\mathcal{Q}^{2} \chi_{0}^{(n)}, \\
\operatorname{Re} \pi_{L}^{(n)}(0, \mathcal{Q}) & =\frac{1}{3} \mathcal{Q}^{2} \chi_{0}^{(n)} v_{F n}^{2}, \\
\operatorname{Re} \pi_{T}^{(n)}(\Omega, 0)=\operatorname{Re} \pi_{L}^{(n)}(\Omega, 0) & =\frac{1}{3} \Omega^{2} \chi_{0}^{(n)} v_{F n}^{2} .
\end{aligned}
$$

In particular, it is instructive to observe that taking the static limit $(\Omega=0$ ) in Eq. (A8) and using the results of Eq. (4.17), we obtain

$$
\frac{1}{\mu(0, \mathcal{Q})}=1-\chi_{0}^{(n)}
$$

which, glanzing at Eq. (4.14), is recognized as the classic Pauli formula for the static magnetic permeability of a degenerate fermion gas. For later reference, it is useful to observe that in the nonrelativistic limit

$$
\chi_{0}^{(n)} \simeq(3.6) \alpha v_{F n}
$$

which follows from Eq. (4.14) by using Eq. (2.17) and setting $\mathcal{P}_{F n} \approx m_{n} v_{F n}$.

For our purposes, the primary quantities of interest are $\pi_{T, L}$, which determine the dispersion relations of the photon modes through Eq. (4.2). If the electron and proton contribution to the self-energy can be neglected and we use Eq. (4.15) for the neutron contribution, the dispersion relations are obtained by solving the equations

$$
\begin{aligned}
\left(\Omega_{T}^{2}-\mathcal{Q}^{2}\right)\left[1-\chi_{0}^{(n)} f_{T}\left(\frac{\Omega_{T}}{v_{F n} \mathcal{Q}}\right)\right] & =0 \\
\left(\Omega_{L}^{2}-\mathcal{Q}^{2}\right)\left[1-\chi_{0}^{(n)} v_{F n}^{2} f_{L}\left(\frac{\Omega_{L}}{v_{F n} \mathcal{Q}}\right)\right] & =0 .
\end{aligned}
$$

The functions $f_{T, L}$ are ploted in Fig. 2. 


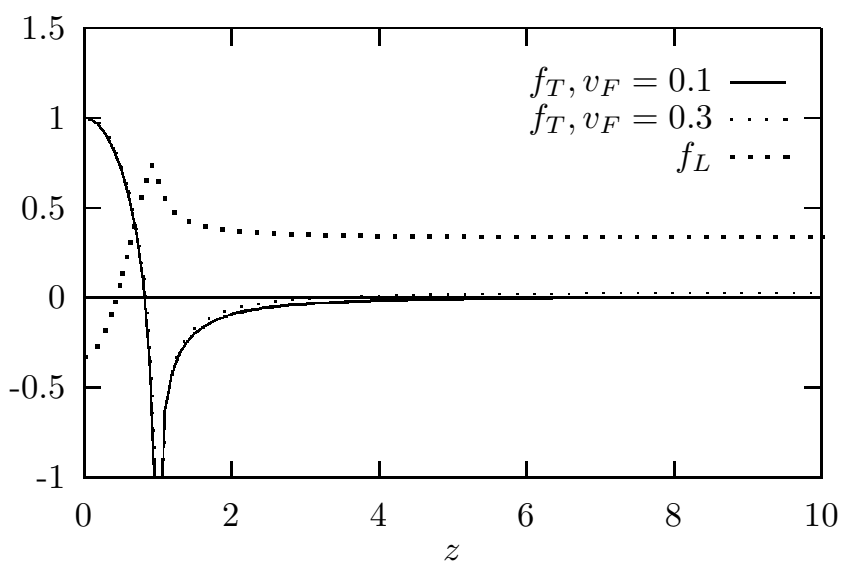

FIG. 2. Plot of the functions $f_{L}$ and $f_{T}$ defined in Eq. (4.16). At $z=1, f_{T}$ becomes infinitely negative. Even for relatively large values of $v_{F}$ the function $f_{T}$ has approximately the same form, and for small values of $v_{F}$ the curves fall almost on top of each other.

As can be seen from the figure, both functions satisfy $f_{T, L} \leq 1$. On the other hand, from Eq. (4.19) we have $\chi_{0}^{(n)}<1$. Therefore Eq. (4.20) has no other solutions than $\Omega_{T, L}=\mathcal{Q}$, so that the photon dispersion relation is not modified by the presence of the background in this case.

\section{Relativistic electron and degenerate neutron gas}

In general, the total background contribution to the transverse and longitudinal components of the photon selfenergy in a matter background is

$$
\pi_{T, L}=\pi_{T, L}^{(e)}+\pi_{T, L}^{(p)}+\pi_{T, L}^{(n)}
$$

Let us neglect for the moment the contribution from the protons, and assume that the electrons can be represented by a relativistic gas and the neutrons by a degenerate non-relativistic gas as considered in Section IV B. In this case $\pi_{T, L}^{(e, n)}$ are given by Eqs. (4.12) and (4.15), respectively.

The dispersion relations for the transverse modes in this case are obtained by solving the equations

$$
q^{2}=\operatorname{Re} \pi_{T}^{(n)}+\operatorname{Re} \pi_{T}^{(e)}
$$

which can be written in the form

$$
q^{2}\left(1-\chi_{0}^{(n)} f_{T}\right)=3 e^{2} \omega_{0 e}^{2} \frac{\Omega}{\mathcal{Q}}\left[\frac{2 \Omega}{\mathcal{Q}}-\frac{q^{2}}{\mathcal{Q}^{2}} \log \left|\frac{\Omega+\mathcal{Q}}{\Omega-\mathcal{Q}}\right|\right] .
$$

While it is not possible to find the general solution to this equation, some useful conclusions can be drawn from it. For example, for values of $\Omega<\mathcal{Q}$, the right hand side of Eq. (4.23) is a positive quantity, while the left hand side is negative since, as we have seen above, $\chi_{0}^{(n)} f_{T}<1$. Therefore, the solution to Eq. (4.23) is such that $\Omega_{T}>\mathcal{Q}$. This implies, in particular, that a particle propagating such a medium cannot emit transverse photons in the form of Cerenkov radiation. Furthermore, since $z_{n}$ is considerably greater than unity for $\Omega>\mathcal{Q}$, the value of $f_{T}\left(z_{n}\right)$ is negligible for that range and therefore the solution to Eq. (4.23) is well approximated by the corresponding solution for the electron background only.

Notice that a possibly different, and erroneous, conclusion would have been obtained if we were to approximate the neutron contribution in Eq. (4.22) by the value in the static limit $\operatorname{Re} \pi_{T}^{(n)}(0, \mathcal{Q})$ given in Eq. (4.17). Then, instead of Eq. (4.23), such an approach leads to the equation

$$
q^{2}=-\mathcal{Q}^{2} \chi_{0}^{(n)}+\operatorname{Re} \pi_{T}^{(e)}
$$

for the dispersion relation, which can have a solution such that $\Omega_{T}<\mathcal{Q}$ depending on the relative size of the two competing terms in the right hand side. 
For the longitudinal modes on the other hand, the dispersion relation is determined by solving the equation

$$
q^{2}\left(1-\chi_{0}^{(n)} v_{F n}^{2} f_{L}\right)=\operatorname{Re} \pi_{L}^{(e)},
$$

with $\operatorname{Re} \pi_{L}^{(e)}$ given by Eq. (4.12). The factor

$$
\frac{\Omega}{2 \mathcal{Q}} \ln \left|\frac{\Omega+\mathcal{Q}}{\Omega-\mathcal{Q}}\right|
$$

which is plotted in Fig. 3, is larger than unity for values of $\Omega / \mathcal{Q}$ larger than about 0.84 . Therefore, there is a small range

$$
0.84 \lesssim \frac{\Omega}{\mathcal{Q}}<1
$$

for which the solution to Eq. (4.25) is such that $\Omega_{L}<\mathcal{Q}$.

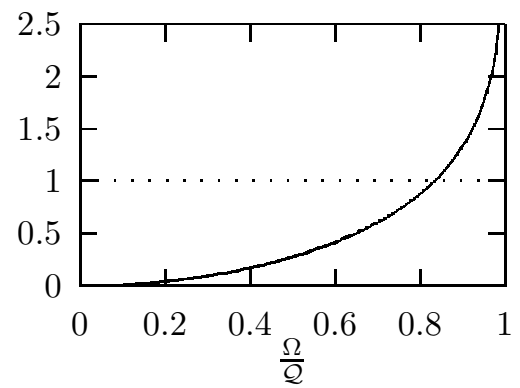

FIG. 3. The factor $\frac{2 \Omega}{\mathcal{Q}} \log \left|\frac{\Omega+\mathcal{Q}}{\Omega-\mathcal{Q}}\right|$ is plotted as a function of $\frac{\Omega}{\mathcal{Q}}$.

Hence, the Cerenkov radiation of longitudinal photons is allowed. For those values of $\Omega / \mathcal{Q}$, the value of $z_{n}$ corresponds to the region in Fig. 2 where the function $f_{L}$ is negligible. Therefore, the neutron component of the background does not play a significant role in the photon dispersion relation in this case either.

\section{Relativistic electron and degenerate neutron and proton gases}

The contribution from the protons can be obtained straightforwardly by substituting Eqs. (3.1) and (3.8) in Eq. (2.35). In the non-relativistic limit, the result can be expressed as

$$
\begin{aligned}
& \operatorname{Re} \pi_{T}^{(p)}=6 e^{2} \omega_{0 p}^{2} g_{T}\left(z_{p}\right)+q^{2} \chi_{0}^{(p)} f_{T}\left(z_{p}\right)+q^{2}\left(\frac{|e|}{m_{p} \kappa_{p}}\right) \chi_{0}^{(p)} h\left(z_{p}\right), \\
& \operatorname{Re} \pi_{L}^{(p)}=12 e^{2} \omega_{0 p}^{2} g_{L}\left(z_{p}\right)+q^{2} \chi_{0}^{(p)} f_{L}\left(z_{p}\right)+q^{2}\left(\frac{|e|}{m_{p} \kappa_{p}}\right) \chi_{0}^{(p)} h\left(z_{p}\right),
\end{aligned}
$$

where, in analogy with Eq. (4.14

$$
\chi_{0}^{(p)} \equiv \frac{\kappa_{p}^{2} m_{p} \mathcal{P}_{F p}}{\pi^{2}}
$$

and

$$
\omega_{0 p}^{2}=\frac{n_{p}}{4 m_{p}},
$$

with $n_{p}$ denoting the proton number density. The functions $f_{T, L}$ are defined in Eq. (4.16), while 


$$
\begin{aligned}
g_{T}(z) & =z^{2}+\frac{1}{2} z\left(1-z^{2}\right) \log \left|\frac{1+z}{1-z}\right|, \\
g_{L}(z) & =\left(z^{2}-\frac{1}{v_{F}^{2}}\right)\left[-1+\frac{1}{2} z \log \left|\frac{1+z}{1-z}\right|\right], \\
h(z) & =1-\frac{1}{6} v_{F}^{2}-\left(1-\frac{1}{2} v_{F}^{2}\right) \frac{1}{2} z \log \left|\frac{1+z}{1-z}\right| .
\end{aligned}
$$

These functions are plotted in Fig. 4
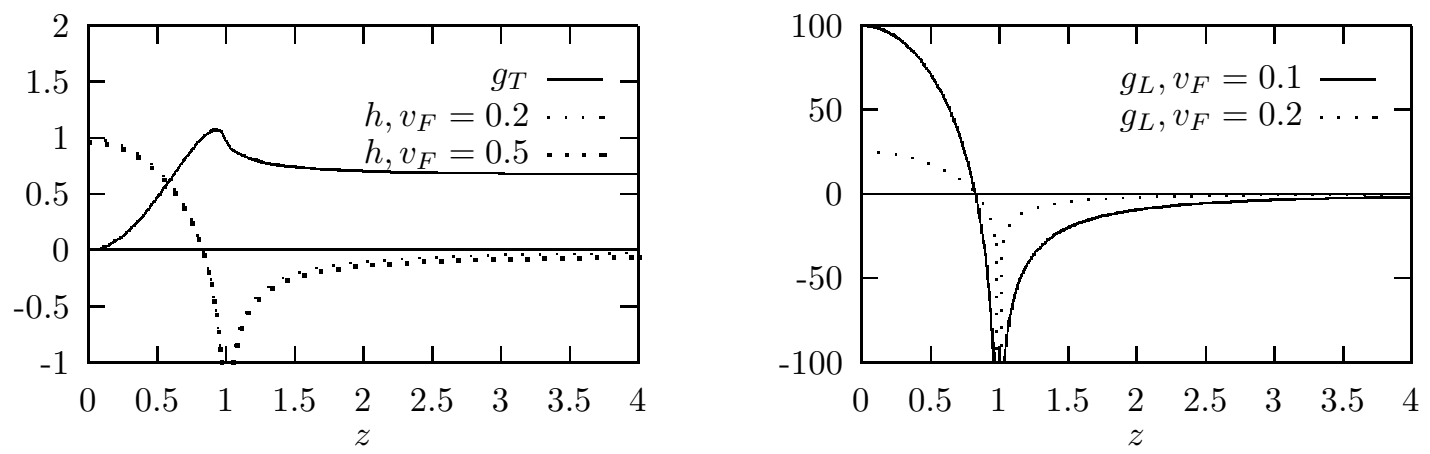

FIG. 4. Plot of the functions $h$ and $g_{T, L}$ for some representative values of $v_{F}$. At $z=1, g_{L}, h$ become infinitely negative. For $z \gg 1$, the functions $h, g_{T}, g_{L}$ have the asymptotic values $\frac{1}{3} v_{F}^{2}, 2 / 3,1 / 3$, respectively.

Let us consider the dispersion relation for the transverse modes. It can be written in the form

$$
q^{2}(1-\chi)=\operatorname{Re} \pi_{T}^{(e)}+6 e^{2} \omega_{0 p}^{2} g_{T}\left(z_{p}\right)
$$

where we have defined

$$
\chi \equiv \chi_{0}^{(n)} f_{T}\left(z_{n}\right)+\chi_{0}^{(p)} f_{T}\left(z_{p}\right)+\chi_{0}^{(p)}\left(\frac{|e|}{m_{p} \kappa_{p}}\right) h\left(z_{p}\right) .
$$

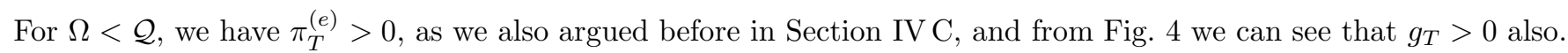
Therefore, the right-hand side of Eq. (4.32) is a positive quantity. On the other hand, since the functions $h$ and $f_{T}$ never become larger than unity, then $\chi<1$ since $\chi_{0}^{(n, p)}$ are considerably smaller than unity (they are of order $\alpha v_{F}$ ). Therefore, Eq. (4.32) has no solution with $\Omega<\mathcal{Q}$. For the opposite regime $\Omega>\mathcal{Q}$, we have $z_{n, p} \gg 1$, and $f_{T}, h, g_{T}$ go to their asymptotic values

$$
\begin{aligned}
h & \rightarrow \frac{1}{3} v_{F}^{2}, \\
f_{T} & \rightarrow \frac{1}{3} v_{F}^{2}, \\
g_{T} & \rightarrow \frac{2}{3} .
\end{aligned}
$$

It then follows that the contribution from the $\chi$ terms in Eq. (4.32) is small. On the right-hand side of that equation, the term $\omega_{0 p}^{2} g_{T}$ from the protons is competing against the corresponding term from the electrons. Since $\omega_{0 e}^{2} \sim T^{2}$ and $n_{e} \sim T^{3}$, where $T$ is the temperature of the electron gas, we have $\omega_{0 e}^{2} \sim n_{p} / T$, assuming that $n_{e} \sim n_{p}$. Then from Eq. (4.30) it follows that

$$
\omega_{0 p}^{2} \approx \frac{T}{m_{p}} \omega_{0 e}^{2},
$$

so that the proton term on the right-hand side of Eq. (4.32) is also small. The equation for the dispersion relation is then well approximated by simply

$$
q^{2}=\pi_{T}^{(e)}
$$


Thus, the nucleon contribution, represented by the $\chi$ and $g_{T}$ terms in Eq. (4.32), may affect some of the details of the transverse dispersion relation for the relativistic electron gas, but it will not modify the general features in an essential way.

The situation can be different for the longitudinal modes. The equation for the dispersion relation can be written in the form

$$
q^{2}\left(1-\chi^{\prime}\right)=\operatorname{Re} \pi_{L}^{(e)}+12 e^{2} \omega_{0 p}^{2} g_{L}\left(z_{p}\right)
$$

where

$$
\chi^{\prime} \equiv \chi_{0}^{(n)} v_{F n}^{2} f_{L}\left(z_{n}\right)+\chi_{0}^{(p)} v_{F p}^{2} f_{L}\left(z_{p}\right)+\chi_{0}^{(p)}\left(\frac{|e|}{m_{p} \kappa_{p}}\right) h\left(z_{p}\right)
$$

The factor $v_{F}^{2}$ in the terms proportional to $f_{L}\left(z_{n, p}\right)$ makes those terms unimportant, while the term proportional to $h$ need not be negligible, although it is small as discussed above. Regarding the terms on the right-hand side of Eq. (4.37), consider first the region $\Omega>\mathcal{Q}$. In that region, we have $z_{p} \gg 1$ and hence $g_{L} \rightarrow 1 / 3$. The same argument as before [Eq. (4.35)] then implies that the proton contribution is not relevant. However, in contrast with the equation for the transverse modes, Eq. (4.37) can have solutions with $\Omega_{L}<\mathcal{Q}$ because $g_{L}$ can be negative and, as we saw in Section IV,$\pi_{L}^{(e)}$ can be negative as well in this range. Furthermore, for values of $\Omega, \mathcal{Q}$ such that $z_{p} \sim 1$, we have $g_{L} \sim-\frac{1}{v_{F}^{2}}$, and this can bring the proton term $\omega_{0 p}^{2} g_{L}$ to be comparable to the electron term $\omega_{0 e}^{2}$. Thus, to summarize the situation for the longitudinal modes, in Eq. (4.37) the term with $f_{L}$ is irrelevant, the term with $h$ can give a small correction, while the term with $g_{L}$ can have an important effect in the dispersion relation. The latter effect can occur in the range of $\Omega, \mathcal{Q}$ such that $z_{p} \sim 1$, if

$$
\frac{\omega_{0 p}^{2}}{v_{F p}^{2}} \sim \omega_{0 e}^{2}
$$

Using Eq. (4.35) this can be translated into the condition $v_{F p}^{2} \sim \frac{T}{m_{p}}$, where $T$ stands for the electron gas temperature.

\section{E. Relativistic electron and classical neutron and proton gases}

For a classical non-relativistic proton gas, the contribution to the self-energy is obtained by inserting the expressions given in Eqs. (3.1) and (3.18 - 3.20) into Eq. (2.35). For the transverse part we thus obtain

$$
\operatorname{Re} \pi_{T}^{(p)}=\frac{|e|^{2} n_{p}}{m_{p}} G_{T}\left(\bar{z}_{p}\right)+\kappa_{p}^{2} n_{p} \beta_{p} q^{2} F_{T}\left(\bar{z}_{p}\right)+\frac{|e| \kappa_{p}}{m_{p}} n_{p} \beta_{p} q^{2} H\left(\bar{z}_{p}\right)
$$

where, to the order that we have calculated,

$$
\begin{array}{r}
G_{T}\left(\bar{z}_{f}\right)= \begin{cases}\bar{z}_{f}^{2} & \left(\bar{z}_{f} \ll 1\right) \\
1+\frac{1}{\bar{z}_{f}^{2}} & \left(\bar{z}_{f} \gg 1\right)\end{cases} \\
F_{T}=H\left(\bar{z}_{f}\right)=\left\{\begin{array}{ll}
1-\bar{z}_{f}^{2} & \left(\bar{z}_{f} \ll 1\right) \\
\bar{v}^{2}-\frac{1}{\bar{z}_{f}^{2}} & \left(\bar{z}_{f} \gg 1\right)
\end{array} .\right.
\end{array}
$$

Similarly, for the longitudinal part,

$$
\operatorname{Re} \pi_{L}^{(p)}=\frac{|e|^{2} n_{p}}{m_{p}} G_{L}\left(\bar{z}_{p}\right)+\frac{\kappa_{p}^{2} n_{p}}{m_{p}} q^{2} F_{L}\left(\bar{z}_{p}\right)+\frac{|e| \kappa_{p}}{m_{p}} n_{p} \beta_{p} q^{2} H\left(\bar{z}_{p}\right),
$$

where

$$
\begin{aligned}
& G_{L}\left(\bar{z}_{f}\right)=\left(\bar{z}_{f}^{2}-\frac{1}{\bar{v}_{f}^{2}}\right) \times\left\{\begin{array}{cc}
-1+\bar{z}_{f}^{2} & \left(\bar{z}_{f} \ll 1\right) \\
\frac{1}{\bar{z}_{f}^{2}}+\frac{3}{\bar{z}_{f}^{4}} & \left(\bar{z}_{f} \gg 1\right)
\end{array}\right. \\
& F_{L}\left(\bar{z}_{f}\right)= \begin{cases}-1+2 \bar{z}_{f}^{2} & \left(\bar{z}_{f} \ll 1\right) \\
1+\frac{2}{\bar{z}_{f}^{2}} & \left(\bar{z}_{f} \gg 1\right) .\end{cases}
\end{aligned}
$$


The formulas for the neutron contribution correspond to setting $|e|=0$ in Eqs. (4.40) and (4.42) and replacing every quantity that refers to the proton by the analogous quantity for the neutron.

Comparing these results with those given in Eq. (4.28) for the degenerate case, it is easily seen that the qualitative features between that case and the present one are similar. In fact, an analysis along the same lines to that carried out in Sections IVC and IVD reveals similar conclusions. In particular, the neutron component does not modify the photon dispersion relations in a significant way. The proton contribution to the transverse component of the self-energy is also unimportant but it can have a noticeable effect for the longitudinal part. This can occur for values of $\Omega<\mathcal{Q}$, but such that $z_{p} \sim 1$. In this case, similarly to the situation for the case of degenerate protons, $G_{L} \sim 1 / \bar{v}_{p}^{2}$, and this brings the proton contribution to $\operatorname{Re} \pi_{L}$ to be of the order of $e^{2} n_{p} \beta_{p}$. Assuming that $n_{p} \sim n_{e}$ and $n_{e} \sim T^{3}$, where $T$ stands for the electron gas temperature, the proton contribution is then of order $e^{2} T^{3} \beta_{p}$. This can compete with the electron contribution, which is of order $e^{2} T^{2}$, if the temperature of both components are comparable. The proton contribution could in fact dominate if the proton gas temperature is smaller than that of the electron gas.

\section{F. Nucleon contribution to the imaginary part}

In general, the concept of a propagating mode with a definite dispersion relation is meaningful provided that the damping rate $\gamma_{T, L}$ is much smaller than $\Omega_{T, L}$ in the solution to Eq. (4.2). Therefore, in the cases in which the nucleon contribution to the real part of the photon self-energy may be substantial, it is imperative to know also the contribution to the imaginary part.

In the general case, the calculation of the nucleon contribution to the imaginary part of the self-energy is cumbersome. Fortunately, for our purposes, in which the small $q$ formulas given in Eqs. (3.1) and (3.2) hold, we can give a simple recipe to determine it. It is given by the so-called Landau rule, whose justification in the present context is provided in Appendix B. The rule is simply that, in the small $q$ limit, the imaginary part of $A_{f}, B_{f}$ can be obtained from Eq. (3.2) by making the replacement $\Omega \rightarrow \Omega+i \epsilon$ in the integrand of those formulas, and taking the imaginary part of the resulting expressions. Thus,

$$
\begin{aligned}
\operatorname{Im} B_{f} & =\frac{\pi \Omega}{2} \int \frac{d^{3} \mathcal{P}}{(2 \pi)^{3}} \delta\left(\Omega-\vec{v}_{\mathcal{P}} \cdot \overrightarrow{\mathcal{Q}}\right) \frac{d}{d \mathcal{E}}\left(f_{f}+f_{\bar{f}}\right), \\
\operatorname{Im} A_{f} & =\frac{\pi \Omega}{2} \int \frac{d^{3} \mathcal{P}}{(2 \pi)^{3}}\left(1-v_{\mathcal{P}}^{2}\right) \delta\left(\Omega-\vec{v}_{\mathcal{P}} \cdot \overrightarrow{\mathcal{Q}}\right) \frac{d}{d \mathcal{E}}\left(f_{f}+f_{\bar{f}}\right), \\
\operatorname{Im} D_{f} & =\frac{1}{2 m_{f}^{2}} \operatorname{Im} A_{f} .
\end{aligned}
$$

Further, the imaginary part of $\pi_{T, L}^{(n, p)}$ is given by formulas analagous to Eq. (2.35), but with the functions $A_{f}, B_{f}, D_{f}$ replaced by their imaginary part, given in Eq. (4.44).

\section{Degenerate case}

For the particular case of the degenerate gas, these formulas reduce to

$$
\begin{aligned}
& \operatorname{Im} B_{f}=-\left(\frac{m_{f} \mathcal{P}_{F f} \gamma_{f}}{8 \pi}\right) z_{f} \theta\left(1-\left|z_{f}\right|\right), \\
& \operatorname{Im} A_{f}=2 m_{f}^{2} \operatorname{Im} D_{f}=\left(1-v_{F f}^{2}\right) \operatorname{Im} B_{f},
\end{aligned}
$$

where $\gamma_{f}$ and $z_{f}$ are defined in Eq. (3.9). These in turn yield,

$$
\begin{aligned}
& \operatorname{Im} \pi_{T}^{(f)}=\pi z_{f} \theta\left(1-\left|z_{f}\right|\right) \gamma_{f}\left\{3 e^{2} \omega_{0 p}^{2}\left(z_{p}^{2}-1\right)+q^{2}\left(\frac{e_{f} \chi_{0}^{(f)}}{2 m_{f} \kappa_{p} \gamma_{f}^{2}}\right)+\frac{1}{2} q^{2} \chi_{0}^{(f)}\left(1-\frac{1}{2} v_{F}^{2} z_{f}^{2}\right)\right\}, \\
& \operatorname{Im} \pi_{L}^{(f)}=\pi z_{f} \theta\left(1-\left|z_{f}\right|\right) \gamma_{f}\left\{-6 e_{f}^{2} \omega_{0 f}^{2}\left(z_{f}^{2}-\frac{1}{v_{F}^{2}}\right)+q^{2}\left(\frac{e_{f} \chi_{0}^{(f)}}{2 m_{f} \kappa_{f}}\right)+\frac{1}{2} q^{2} \chi_{0}^{(f)} v_{F}^{2}\left(z_{f}^{2}-1\right)\right\} .
\end{aligned}
$$


The formulas in Eq. (4.44) can be evaluated explicitly also for the classical non-relativistic case. With $\bar{z}_{f}$ as defined in Eq. (3.21), the results can be expressed in the form

$$
\begin{aligned}
& \operatorname{Im} B_{f}=-\frac{1}{4} \sqrt{\frac{\pi}{2}} \beta_{f} n_{f} \bar{z}_{f} e^{-\frac{1}{2} \bar{z}_{f}^{2}} \\
& \operatorname{Im} A_{f}=2 m_{f}^{2} \operatorname{Im} D_{f}=\operatorname{Im} B_{f}\left[1-\bar{v}_{f}^{2}\left(2+\bar{z}_{f}^{2}\right)\right]
\end{aligned}
$$

which imply

$$
\begin{aligned}
& \operatorname{Im} \pi_{T}^{(f)}=\sqrt{\frac{\pi}{2}} \beta_{f} n_{f} \bar{z}_{f} e^{-\frac{1}{2} \bar{z}_{f}^{2}}\left\{-e_{f}^{2} \bar{v}_{f}^{2}+\frac{e_{f} \kappa_{f}}{m_{f}} q^{2}\left(1-\bar{v}_{f}^{2} \bar{z}_{f}^{2}\right)+\kappa_{f}^{2} q^{2}\left(1-\bar{v}_{f}^{2} \bar{z}_{f}^{2}\right)\right\}, \\
& \operatorname{Im} \pi_{L}^{(f)}=\sqrt{\frac{\pi}{2}} \beta_{f} n_{f} \bar{z}_{f} e^{-\frac{1}{2} \bar{z}_{f}^{2}}\left\{e_{f}^{2}\left(1-\bar{v}_{f}^{2} \bar{z}_{f}^{2}\right)+\frac{e_{f} \kappa_{f}}{m_{f}} q^{2}\left(1-\bar{v}_{f}^{2} \bar{z}_{f}^{2}\right)-2 \kappa_{f}^{2} q^{2} \bar{v}_{f}^{2}\right\} .
\end{aligned}
$$

As usual, these formulas allow us to obtain the results for the proton and neutron separately, by setting $e_{f}$ and $\kappa_{f}$ to the appropriate value in each case.

\section{CONCLUSIONS}

In this work we have calculated the 1-loop contribution to the photon self-energy in a background composed of electrons and nucleons, taking into account the anomalous magnetic moment couplings of the nucleons to the photon. The generic contribution from a fermion to the real part of the photon self-energy is given by the formulas in Eq. (2.35), in terms of the quantities that we have denoted by $A_{f}, B_{f}, D_{f}$. The explicit evaluation of these three functions is carried out in Section III for various limiting cases of interest. The results obtained there were applied in Section IV] to consider the effect of including the nucleon contribution to the photon dispersion relation in a plasma. For concreteness, we considered the case of a relativistic electron gas, superimposed on a nonrelativistic nucleon background, which we considered to be degenerate or classical in separate cases. The results of that exercise indicate that the magnetic moment couplings of the nucleons do not have a significant effect on the photon dispersion relations. Hence the neutrons, which only have these type of coupling, do not seem to play a siginificant role in the determination of the photon dispersion relations in such media. For the protons, the normal couplings do not have a significant effect on the transverse modes either, but they can have an impact on the longitudinal dispersion relation in some circumstances. As shown in Sections IVD and IVE, for values of the photon momentum such that $\Omega \sim \bar{v}_{p} \mathcal{Q}$, where $\bar{v}_{p}$ is a typical velocity of the protons in the gas, the proton contribution to the longitudinal component of the photon self-energy can be of the same order as that of the electrons. Of course we have reached these conclusions by considering in detail the application of the general formulas to some particular idealized situations. In any case, the formulas that we have provided in this work open the way to similar studies in other possible settings.

This work has been partially supported by the U.S. National Science Foundation Grant PHY-9600924 (JFN) and by Grant No. DGAPA-IN100694 at the Universidad Nacional Autónoma de México (JCD).

\section{APPENDIX A: DIELECTRIC FUNCTION}

Although the properties of photons propagating through the medium can be deduced from the knowledge of the photon self-energy directly, it is useful to translate those results into the language of macroscopic electrodynamics. The transverse and longitudinal components of the dielectric constant of the medium are introduced by writing the induced current, in the rest frame of the medium, as

$$
\vec{\jmath}^{(i n d)}=i \Omega\left[\left(1-\epsilon_{l}\right) \vec{E}_{l}+\left(1-\epsilon_{t}\right) \vec{E}_{t}+i \epsilon_{p} \hat{\mathcal{Q}} \times \vec{E}\right],
$$

where the longitudinal and transverse components of the electric field are defined by

$$
\begin{aligned}
& \vec{E}_{l}=\hat{\mathcal{Q}}(\hat{\mathcal{Q}} \cdot \vec{E}), \\
& \vec{E}_{t}=\vec{E}-\vec{E}_{l} .
\end{aligned}
$$


This is the most general form of the induced current, involving terms that are linear in the field, and subject only to the assumption of isotropy. On the other hand, by comparing Maxwell's equations with the effective field equation Eq. (2.1), it follows that the induced current is given by

$$
j_{\mu}^{(i n d)}=-\pi_{\mu \nu} A^{\nu}
$$

which can be written as in Eq. (A1) with

$$
\begin{aligned}
1-\epsilon_{t} & =\frac{\pi_{T}}{\Omega^{2}}, \\
1-\epsilon_{l} & =\frac{\pi_{L}}{q^{2}}, \\
\epsilon_{p} & =\frac{\pi_{P}}{\Omega^{2}} .
\end{aligned}
$$

Alternatively to $\epsilon_{t, l}$, the dielectric and magnetic permeability functions $\epsilon, \mu$ are introduced by writing the induced current in the equivalent form

$$
\vec{\jmath}^{(i n d)}=i \Omega\left[(1-\epsilon) \vec{E}+i\left(1-\frac{1}{\mu}\right) \vec{q} \times \vec{B}+i \epsilon_{p} \hat{\mathcal{Q}} \times \vec{E}\right],
$$

where

$$
\vec{B}=\frac{1}{\Omega} \vec{q} \times \vec{E}
$$

Comparing Eqs. (A1) and (A5), the relations

$$
\begin{aligned}
\epsilon & =\epsilon_{l} \\
\frac{1}{\mu} & =1+\frac{\Omega^{2}}{\mathcal{Q}^{2}}\left(\epsilon_{l}-\epsilon_{t}\right)
\end{aligned}
$$

then follow, and in particular

$$
\frac{1}{\mu}=1+\frac{1}{\mathcal{Q}^{2}}\left(\pi_{T}-\frac{\Omega^{2}}{q^{2}} \pi_{L}\right)
$$

\section{APPENDIX B: IMAGINARY PART}

From Eq. (2.3), it follows that

$$
\operatorname{Im} \pi_{\mu \nu}^{(e f f)}=\varepsilon(q \cdot v) \operatorname{Im} \pi_{\mu \nu},
$$

where $\operatorname{Im} \pi_{\mu \nu}$ can be determined in terms of the components of the self-energy matrix by either one of the formulas given in Eq. (2.5). However, as already mentioned in Section III, it is much easier in practice to calculate $\pi_{12 \mu \nu}$ than it is to calculate $\operatorname{Im} \pi_{11 \mu \nu}$, although the procedure in both cases is similar. As an illustration, we outline below the main steps of the calculation in terms of $\pi_{12 \mu \nu}$, although we have verified explicitly that the same result is obtained using the alternate form in terms of $\operatorname{Im} \pi_{11 \mu \nu}$. We start by considering the contribution from the electron loop, and then generalize the result to the case of the nucleons afterwards.

From Fig. 囵.

$$
i \pi_{12 \mu \nu}^{(e)}=(-1)(-i e)(i e) \operatorname{Tr} \int \frac{d^{4} p}{(2 \pi)^{4}} \gamma_{\mu} i S_{F 12}^{(e)}\left(p^{\prime}\right) \gamma \nu i S_{F 21}^{(e)}(p)
$$

where

$$
p^{\prime}=p+q .
$$

After substituting the propagators given in Eq. (2.10) we use the relation 


$$
\begin{aligned}
{\left[\eta_{e}\left(p^{\prime}\right)-\theta\left(-p^{\prime} \cdot v\right)\right]\left[\eta_{e}(p)-\theta(p \cdot v)\right]=} & -n(x)\left[\eta_{e}(p) \varepsilon\left(p^{\prime} \cdot v\right)-\eta_{e}\left(p^{\prime}\right) \varepsilon(p \cdot v)\right. \\
& \left.+\theta(p \cdot v) \theta\left(-p^{\prime} \cdot v\right)-\theta(-p \cdot v) \theta\left(p^{\prime} \cdot v\right)\right],
\end{aligned}
$$

which is proven by using the identities

$$
\begin{aligned}
\eta_{e}(p)-\theta(p \cdot v) & =-e^{y_{e}} n_{F}\left(y_{e}\right) \varepsilon(p \cdot v), \\
\eta_{e}(p)-\theta(-p \cdot v) & =n_{F}\left(y_{e}\right) \varepsilon(p \cdot v),
\end{aligned}
$$

together with

$$
e^{y_{e}} n_{F}\left(y_{e}\right) n_{F}\left(y_{e}^{\prime}\right)=n_{\gamma}(x)\left(n_{F}\left(y_{e}\right)-n_{F}\left(y_{e}^{\prime}\right)\right) .
$$

In Eq. (B6) we have defined

$$
y_{e}^{\prime}=p^{\prime}-\alpha_{e},
$$

while $x$ and $y_{e}$ are defined in Eqs. (2.8) and (2.13), respectively, and we have used the fact that $y_{e}^{\prime}=y_{e}+x$. In this way we obtain

$$
\begin{aligned}
i \pi_{12 \mu \nu}^{(e)}= & 16 \pi^{2} e^{2} n_{\gamma}(x) \int \frac{d^{4} p}{(2 \pi)^{4}} L_{\mu \nu} \delta\left(p^{2}-m_{e}^{2}\right) \delta\left(p^{2}-m_{e}^{2}\right) \\
& \times\left\{\eta_{e}(p) \varepsilon\left(p^{\prime} \cdot v\right)-\eta_{e}\left(p^{\prime}\right) \varepsilon(p \cdot v)+\theta(p \cdot v) \theta\left(-p^{\prime} \cdot v\right)-\theta(-p \cdot v) \theta\left(p^{\prime} \cdot v\right)\right\} .
\end{aligned}
$$

Making the change of variable $p+q \rightarrow p$ in the second and fourth terms in braces, Eq. (2.9) then yields

$$
\begin{aligned}
\operatorname{Im} \pi_{e \mu \nu}^{(e f f)}= & 4 \pi e^{2} \int \frac{d^{4} p}{(2 \pi)^{4}}\left\{L_{\mu \nu} \delta\left[(p+q)^{2}-m_{e}^{2}\right)\right] \delta\left(p^{2}-m_{e}^{2}\right) \\
& \left.\times\left[\eta_{e}(p) \varepsilon((p+q) \cdot v)+\theta(p \cdot v) \theta(-(p+q) \cdot v)\right]+(q \rightarrow-q)\right\},
\end{aligned}
$$

for the electron contribution to the imaginary part of $\pi_{\mu \nu}^{(e f f)}$. After doing the integral over $p_{0}$, this reduces to

$$
\begin{aligned}
\operatorname{Im} \pi_{e \mu \nu}^{(e f f)}= & 4 \pi e^{2} \int \frac{d^{3} \mathcal{P}}{(2 \pi)^{3} 2 \mathcal{E}}\left\{L_{\mu \nu} \delta\left[(p+q)^{2}-m_{e}^{2}\right]\right. \\
& \left.\times\left[\theta((p+q) \cdot v)\left(f_{e}+f_{\bar{e}}\right)+\theta(-(p+q) \cdot v)\left(1-f_{e}-f_{\bar{e}}\right)\right]-(q \rightarrow-q)\right\} .
\end{aligned}
$$

Decomposing $\pi_{e \mu \nu}^{(e f f)}$ according to Eq. (2.3), Eq. (B10) then implies

$$
\begin{aligned}
& \operatorname{Im} \pi_{T}^{(e)}=-2 e^{2}\left(\operatorname{Im} A_{e}+\frac{q^{2}}{\mathcal{Q}^{2}} \operatorname{Im} B_{e}\right), \\
& \operatorname{Im} \pi_{L}^{(e)}=4 e^{2} \frac{q^{2}}{\mathcal{Q}^{2}} \operatorname{Im} B_{e}
\end{aligned}
$$

where

$$
\begin{aligned}
\operatorname{Im} A_{e}= & \pi \int \frac{d^{3} \mathcal{P}}{(2 \pi)^{3} 2 \mathcal{E}}\left\{\left(2 m_{e}^{2}-2 p \cdot q\right) \delta\left[(p+q)^{2}-m_{e}^{2}\right]\right. \\
& \left.\times\left[\theta((p+q) \cdot v)\left(f_{e}+f_{\bar{e}}\right)+\theta(-(p+q) \cdot v)\left(1-f_{e}-f_{\bar{e}}\right)\right]-(q \rightarrow-q)\right\}, \\
\operatorname{Im} B_{e}= & \pi \int \frac{d^{3} \mathcal{P}}{(2 \pi)^{3} 2 \mathcal{E}}\left\{\left[2(p \cdot v)^{2}+2(p \cdot v)(q \cdot v)-p \cdot q\right] \delta\left[(p+q)^{2}-m_{e}^{2}\right]\right. \\
& \left.\times\left[\theta((p+q) \cdot v)\left(f_{e}+f_{\bar{e}}\right)+\theta(-(p+q) \cdot v)\left(1-f_{e}-f_{\bar{e}}\right)\right]-(q \rightarrow-q)\right\} .
\end{aligned}
$$

The formulas in Eq. (B12) complement those in Eq. (2.32), which hold for the real part. The counterpart of the approximate formulas given in Eq. (3.2) for the small $q$ regime are obtained as follows. For $q \ll\langle\mathcal{E}\rangle$, we can use

$$
\theta[-(p+q) \cdot v] \delta\left[(p+q)^{2}-m_{e}^{2}\right]=0
$$


to discard some terms in Eq. (B12). The terms discarded in this way are the standard vacuum terms as well as the temperature corrections to them, which they are relevant only for $q>2 m_{e}$ and physically correspond to the creation of electron-positron pairs. The surviving terms can be written in the form

$$
\begin{aligned}
\operatorname{Im} B_{e}= & \pi \int \frac{d^{3} \mathcal{P}}{(2 \pi)^{3}}\left\{\frac{\left(2 \mathcal{E}_{\overrightarrow{\mathcal{P}}}^{2}+\mathcal{E}_{\overrightarrow{\mathcal{P}}} \Omega+\overrightarrow{\mathcal{P}} \cdot \vec{q}\right)}{4 \mathcal{E}_{\overrightarrow{\mathcal{P}}} \mathcal{E}_{\overrightarrow{\mathcal{P}}+\vec{q}}} \delta\left(\Omega+\mathcal{E}_{\overrightarrow{\mathcal{P}}}-\mathcal{E}_{\overrightarrow{\mathcal{P}}+\vec{q}}\right)\left(f_{e}+f_{\bar{e}}\right)-(q \rightarrow-q)\right\}, \\
\operatorname{Im} A_{e}= & \operatorname{Im} B_{e} \\
& +\pi \int \frac{d^{3} \mathcal{P}}{(2 \pi)^{3}}\left\{\frac{\left(2 \overrightarrow{\mathcal{P}}^{2}-3 \mathcal{E}_{\overrightarrow{\mathcal{P}}} \Omega+\overrightarrow{\mathcal{P}} \cdot \vec{q}\right)}{4 \mathcal{E}_{\overrightarrow{\mathcal{P}}} \mathcal{E}_{\overrightarrow{\mathcal{P}}+\vec{q}}} \delta\left(\Omega+\mathcal{E}_{\overrightarrow{\mathcal{P}}}-\mathcal{E}_{\overrightarrow{\mathcal{P}}+\vec{q}}\right)\left(f_{e}+f_{\bar{e}}\right)-(q \rightarrow-q)\right\},
\end{aligned}
$$

where we have made explicit the dependence of the energy on the momentum variable. Making the change of variable $\overrightarrow{\mathcal{P}} \rightarrow \overrightarrow{\mathcal{P}}-\frac{1}{2} \vec{q}$ and expanding the integrand up to terms that are linear in $q$, we obtain

$$
\begin{aligned}
\operatorname{Im} B_{e} & =-\frac{\pi}{2} \Omega \int \frac{d^{3} \mathcal{P}}{(2 \pi)^{3}} \delta(\Omega-\overrightarrow{\mathcal{P}} \cdot \vec{q}) \frac{d}{d \mathcal{E}}\left(f_{e}+f_{\bar{e}}\right), \\
\operatorname{Im} A_{e} & =\operatorname{Im} B_{e}+\frac{\pi}{2} \Omega \int \frac{d^{3} \mathcal{P}}{(2 \pi)^{3}} v_{\mathcal{P}}^{2} \delta(\Omega-\overrightarrow{\mathcal{P}} \cdot \vec{q}) \frac{d}{d \mathcal{E}}\left(f_{e}+f_{\bar{e}}\right) .
\end{aligned}
$$

The results given in Eq. (B15), together with Eq. (B11), provide the justification of the rule stated in Section IVF, as can be immediately confirmed by applying it to determine the imaginary part of $\pi_{T, L}^{(e)}$ from the formulas in Eq. (4.10) for the real part of the same quantities. The same arguments given here also to apply to the nucleon contribution, the only difference being that in the formula corresponding to Eq. (B10), the factor $e^{2} L_{\mu \nu}$ is replaced by

$$
e_{f}^{2} L_{\mu \nu}+\kappa_{f}^{2} M_{\mu \nu}+2 e_{f} \kappa_{f} m_{f} q^{2} \tilde{g}_{\mu \nu} .
$$

By mimicking the steps that lead to Eq. (2.35), we obtain similarly looking formulas for $\operatorname{Im} \pi_{T, L}^{(f)}$, but with the functions $A_{f}, B_{f}, D_{f}$ replaced by their imaginary parts, which in the end yield the results quoted in Section IV F.

[1] For a review see, for example, N. P. Landsman and Ch. G. van Weert, Phys. Rep. 145, 141 (1987).

[2] H. A. Weldon, Phys. Rev. D26, 1394 (1982). See also, E. Braaten and D. Segel, Phys. Rev. D48, 1478 (1993).

[3] H. A. Weldon, Phys. Rev. D26, 2789 (1982).

[4] H. A. Weldon, Phys. Rev. D28, 2007 (1983).

[5] E. M. Lifshitz and L. P. Pitaevskii, "Physical Kinetics", Course of Theoretical Physics Volume 10, (Pergamon Press, New York 1981), p. 133.

[6] V. N. Tsytovich, J. Exptl. Theoret. Phys. (U.S.S.R) 40, 1775 (June 1961) [Sov. Phys. JETP 13, 1249 (1961)].

[7] V. P. Silin, Zh. Eksp. Teor. Fiz. 38, 1577 (1960) [Sov. Phys. JETP 11, 1136 (1960)].

[8] See, for example, Ref. [5], p. 130.

[9] A. L. Fetter and J. D. Walecka, "Quantum Theory of Many-Particle Systems" (McGraw-Hill, New York, 1971$)$ p. 49.

[10] S. Mohanty and M. K. Samal, Phys. Rev. Lett. 77, 806 (1996).

[11] Georg G. Raffelt, Phys. Rev. Lett. 79, 773 (1997).

[12] José F. Nieves, Phys. Rev. D42, 4123 (1990); ibid.49, 3067(E) (1994).

[13] José F. Nieves and P. B. Pal, Phys. Rev. D39, 652 (1989) and ibid.40, 2148E (1989); ibid.40, 1350 (1989).

[14] J. C. D'Olivo, José F. Nieves and P. B. Pal, Phys. Rev. D40, 3679 (1989).

[15] J. C. D'Olivo, José F. Nieves, "Nucleon contribution to the neutrino electromagnetic vertex in matter", UPR preprint.

[16] See, for example, Ref. 胢, p. 132. 